\title{
Padrões morfológicos na venação alar de espécies de Tetragona Lepeletier \& Serville, 1828 do grupo clavipes (Hymenoptera: Apidae: Meliponini)
}

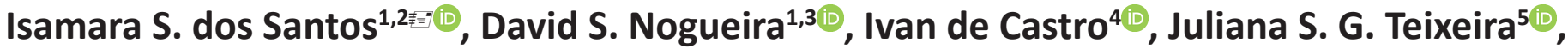 \\ Geusa S. de Freitas ${ }^{4}$, Marcio L. Oliveira ${ }^{1 \oplus}$
}

${ }^{1}$ Instituto Nacional de Pesquisas da Amazônia (INPA), Manaus, Amazonas, Brasil. 2Universidade Federal do Amazonas (UFAM), Manaus, Amazonas, Brasil. ${ }^{3}$ Instituto Federal de Educação, Ciência e Tecnologia do Amazonas (IFAM), São Gabriel da Cachoeira, Amazonas, Brasil. ${ }^{4}$ Faculdade de Medicina de Ribeirão Preto, Universidade de São Paulo (USP), Ribeirão Preto, São Paulo, Brasil. 5 Instituto Tecnológico Vale (ITV), Belém, Pará, Brasil.

拝=”Corresponding author: isabranches.ib@gmail.com

Edited by: Pedro G. B. S. Dias

Received: August 09, 2021. Accepted: November 02, 2021. Published: November 19, 2021.

Morphological patterns of wing venation in Tetragona Lepeletier \& Serville, 1828 species of the clavipes group (Hymenoptera: Apidae: Meliponini)

Abstract. Tetragona Lepeletier \& Serville, 1828 (Hymenoptera: Apidae) is a genus of stingless bees widely distributed in Brazil. It has 15 species distributed in the Neotropics, from Mexico to Uruguay, nine of which are found in Brazil. However, Tetragona elongata (Lepeletier \& Serville, 1828), a species known only from the Southeast region and which had been synonymized with Tetragona clavipes (Fabricius, 1804), was revalidated without any justification. The aim of this study was to test whether the morphometrics analysis of the wings is efficient in the diagnosis of the species of this genus, in addition to testing the validity of the revalidation mentioned above. This technique was applied by accessing the right forewings of 660 workers of T. clavipes, T. elongata e T. quadrangula (Lepeletier, 1836), from five Brazilian collections. For the geometric morphometric analysis, 12 landmarks were selected. The software MorphoJ version 1.6 was used to do Discriminant Function analysis (1000 replications) and Canonical Variation Analysis (CVA). Between T. clavipes and T. elongata, there was a 100\% variance between species (canonical variation analysis), suggesting that it may be an indication of speciation. Even though T. elongata has been revalidated, it still has overlapped with T. clavipes, which indicates to be the same species. Taxonomic studies are needed to synonymize them.

Keywords: Shape variation, species, stingless bee, taxonomy, wing venation.

Tetragona Lepeletier \& Serville, 1828 (Hymenoptera: Apidae: Meliponini), possui espécies que estão distribuídas do México ao Uruguai, sendo nove com ocorrência para o Brasil: Tetragona clavipes (Fabricius, 1804), Tetragona dorsalis (Smith, 1854), Tetragona elongata (Lepeletier \& Serville, 1828), Tetragona essequiboensis (Schwarz, 1940), Tetragona goettei (Friese, 1900), Tetragona handlirschii (Friese, 1900), Tetragona kaieteurensis (Schwarz, 1938), Tetragona truncata Moure, 1971 e Tetragona quadrangula (Lepeletier, 1836). Sendo que Pedro (2014) revalidou T. elongata, espécie de ocorrência conhecida apenas para a região Sudeste, a qual havia sido sinonimizada sob $T$. clavipes por Spinola (1840). Tetragona clavipes faz parte do grupo clavipes e possui cinco espécies similares morfologicamente ( $T$. clavipes, $T$. elongata, T. quadrangula, T. perangulata (Cockerell, 1917) e T. dissecta Moure, 2000).

A espécie T. clavipes foi descrita por Fabricius (1804) e é uma espécie nativa da América do Sul, sendo encontrada da Colômbia até Uruguai, passando pela Bolívia, Brasil, Guiana, Paraguai, Peru e Suriname. No Brasil ocorre nos biomas Amazônia, Cerrado, Mata Atlântica, Pantanal e Pampa (Camargo \& Pedro 2013) onde faz seus ninhos em ocos de árvores. Possui colônias muito populosas, de comportamento territorialista e bastante defensivo, com abelhas-guardas que mordem e depositam resina em quem se aproxima ou interfere em seu ninho (Kerr et al. 1967; Oliveira et al. 2013; Duarte et al. 2016). Morfologicamente, as operárias de $T$. clavipes possuem corbícula bem desenvolvida, a metade apical das asas mais hialinas e possuem o corpo com 6 a $8 \mathrm{~mm}$ de comprimento. A espécie é popularmente conhecida como "borá" e além de possuir muitas variações morfológicas, como a coloração dos pelos da fronte e o padrão de altura e inclinação da mancha paraocular, que confundem a sua real identificação e separação de outras espécies (Nogueira et al. 2020; 2021).

A separação de espécies próximas pode ser elucidada pela técnica de morfometria geométrica, que consiste em um estudo estatístico da variação da forma em relação a fatores causais, que quantifica as formas biológicas e faz inferências sobre causas que levam às diferenças de forma (Bookstein 1991; Monteiro \& Reis 1999). A confusão taxonômica envolvendo as diferentes espécies de Tetragona pode ser avaliada por diferentes técnicas. Uma delas é avaliar o quanto variações morfológicas sutis nas formas de determinadas estruturas dos insetos, como o padrão da venação das asas, por exemplo, podem ser estatisticamente diferentes entre os organismos em questão, ajudando a solucionar questões complexas como diferenciação de espécies próximas filogeneticamente. Essa técnica tem sido uma ferramenta bem-sucedida no estudo com abelhas "sem ferrão", que sugerem que diferentes padrões na venação alar das asas anteriores ajudam a identificar, discriminar e caracterizar gêneros, espécies e até mesmo raças de abelhas (Francoy et. al. 2006; Francoy et. al. 2008; Francoy et. al. 2009).

O objetivo deste estudo foi realizar análises morfométricas da venação alar das espécies de Tetragona do grupo clavipes na tentativa de esclarecer a separação ou sobreposição entre elas, distinguindo 
morfótipos identificados de diferentes regiões do Brasil. Com isso, objetivou-se também testar a validade da revalidação proposta por Pedro (2014).

Foram estudadas 660 espécimes de operárias, preservadas em via seca, da Coleção de Invertebrados do Instituto Nacional de Pesquisas da Amazônia (INPA, Manaus, Amazonas; $n=395$ ), além de material obtido por empréstimo de outras coleções, como o Museu Paraense Emílio Goeldi (MPEG, Belém, Pará; $n=87$ ), Faculdade de Medicina de Ribeirão Preto (FMRP-USP, Ribeirão Preto, São Paulo; n=61), Coleção Prof. J.M.F. Camargo, Faculdade de Filosofia, Ciências e Letras de Ribeirão Preto (RPSP-USP, Ribeirão Preto, São Paulo, n=23), Coleção Entomológica Paulo Nogueira-Neto (CEPANN-IB/USP, São Paulo, São Paulo; n=41) e Universidade Federal de Viçosa (UFV, Viçosa, Minas Gerais; $n=76$ ).

Para a confecção das lâminas permanentes, foi removida a asa anterior direita de operárias de T. clavipes $(n=219), T$. elongata $(n=414)$ e $T$. quadrangula $(n=27)$. Cada asa foi extraída manualmente e imersa em Acetato de Butila, sendo fixada em uma lamínula com Bálsamo do Canadá. Foram utilizadas lamínulas (24 x $24 \mathrm{~mm})$, que foram divididas em quatro partes com o auxílio de estilete. As lâminas foram deixadas em estufa em $40^{\circ} \mathrm{C}$ até a secagem. Todo esse processo foi feito pela mesma pessoa. Para a captura das imagens foi utilizado o estereomicroscópio Leica M205A acoplado à câmera Leica DMC4500 com o software Leica Application Suite V4.1. Para análises da morfometria geométrica e escolha de 12 marcos anatômicos (Fig. 1) foram utilizados os softwares tpsUtil versão 1.64, tpsDig versão 2.22 tpsRelw versão 1.46. Foi utilizado o software MorphoJ versão 1.6 para realização das análises de Função Discriminante (com 1000 replicações), que examina a separação entre dois grupos observados, e Análises das Variáveis Canônicas (CVA), que é um método utilizado para encontrar características na forma que melhor difere entre os múltiplos grupos de amostras analisadas. Para as análises foram utilizadas três espécies do grupo clavipes ( $T$. clavipes, T. elongata e T. quadrangula) (Fig. 2A, 2B e 2C), com os seguintes critérios: "Região", com 52 indivíduos do Centro-Oeste, 20 do Nordeste, 167 do Norte, 348 do Sudeste e 73 do Sul; e "Espécie" com 219 indivíduos de T. clavipes, 414 de T. elongata e 27 de T. quadrangula.

Foi realizada uma análise de Função Discriminante com o critério "Estados" com T. clavipes do Pará e T. elongata do Paraná (102 e 73 espécimes, respectivamente); e uma segunda análise com o classificador "Espécie", com apenas T. clavipes e T. elongata (236 e 412 espécimes, respectivamente).

Com os resultados obtidos a partir da CVA com a variável "Região", verificou-se uma sobreposição das espécies encontradas em todas as regiões estudadas. Na CVA com a variável "Espécie", verificou-se que $T$. clavipes e $T$. elongata se separam parcialmente no gráfico (Fig. 2D), formando dois conjuntos, com uma sobreposição entre as espécies que é suportada com 93,22\%.

A análise isolada das amostras de populações distantes, como dos estados do Pará e Paraná, desconsiderando todas as populações geograficamente intermediárias, evidencia uma separação maior entre os grupos dos indivíduos tratados como T. clavipes e T. elongata usando os critérios de "Espécie" e "Estado", com taxa de sobreposição abaixo de $15 \%$ na Função Discriminante.

Quando avaliamos a tabela de validação cruzada com as espécies T. clavipes e T. elongata (Tab. 1) no critério "Espécie", percebemos que $84,02 \%$ de indivíduos de $T$. clavipes foram classificados de maneira correta, assim como $89,86 \%$ de T. elongata ( $p<0,0001)$, além disso, 35 indivíduos de $T$. clavipes foram classificados como T. elongata $(n=219)$ e 40 de T. elongata foram identificados como T. clavipes $(n=414)$ (Tab. 1).

Tabela 1. Validação cruzada da Análise Discriminante com o classificador "Espécie", entre Tetragona clavipes (Fabricius, 1804) e Tetragona elongata (Lepeletier \& Serville, 1828).

\begin{tabular}{cccc}
\hline Espécie & Tetragona clavipes & Tetragona elongata & Total \\
\hline Tetragona clavipes & 184 & 35 & 219 \\
Tetragona elongata & 42 & 372 & 414 \\
\hline
\end{tabular}

A variação na venação alar encontrada não foi significante para a separação das espécies, pois houve sobreposição entre pontos encontrados nos gráficos ( $p<0,0001$ ) (Fig. 2D e 2E). Na análise das variações canônicas com o classificador "Espécie" (Fig. 2D), houve uma sobreposição de T. clavipes e T. elongata, com explicação de $100 \%$ entre espécies, sugerindo que as pequenas mudanças morfométricas encontradas não foram significantes para distinguir as espécies através da venação alar. O conjunto de $T$. quadrangula não mostrou separação clara em relação aos de $T$. clavipes $+T$. elongata, que pode ser inferido pelo viés da baixa amostra disponível nesta análise ou que sua asa seja muito similar às das outras, sugerindo ainda alguma dúvida em relação à sua validade taxonômica.

Esse estudo se mostrou promissor no sentido do entendimento sobre a relação entre as espécies de Tetragona do grupo clavipes, pois indicou que T. clavipes e T. elongata podem corresponder a uma única espécie. Além disso, estudos mais aprofundados de morfologia e que incluam outras técnicas auxiliares podem ser interessantes para reforçar essa hipótese de sinonimização taxonômica.

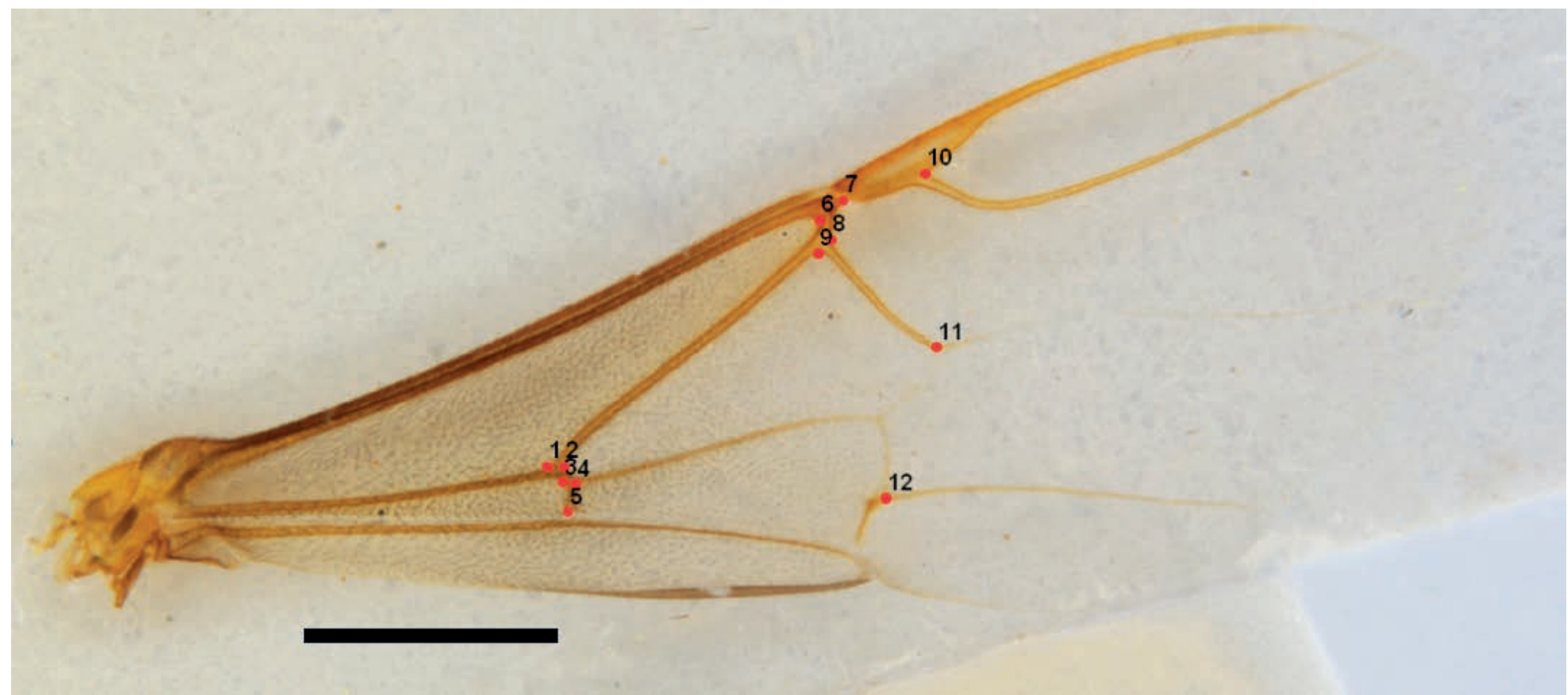

Figura 1. Marcos anatômicos utilizados nas asas de Tetragona Lepeletier \& Serville, 1828 no software tpsDig. Escala: 1 mm. 


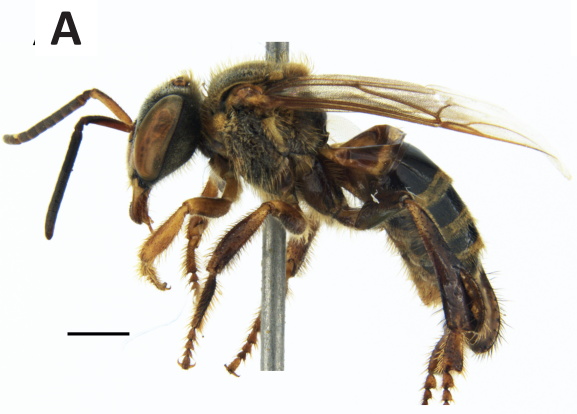

B
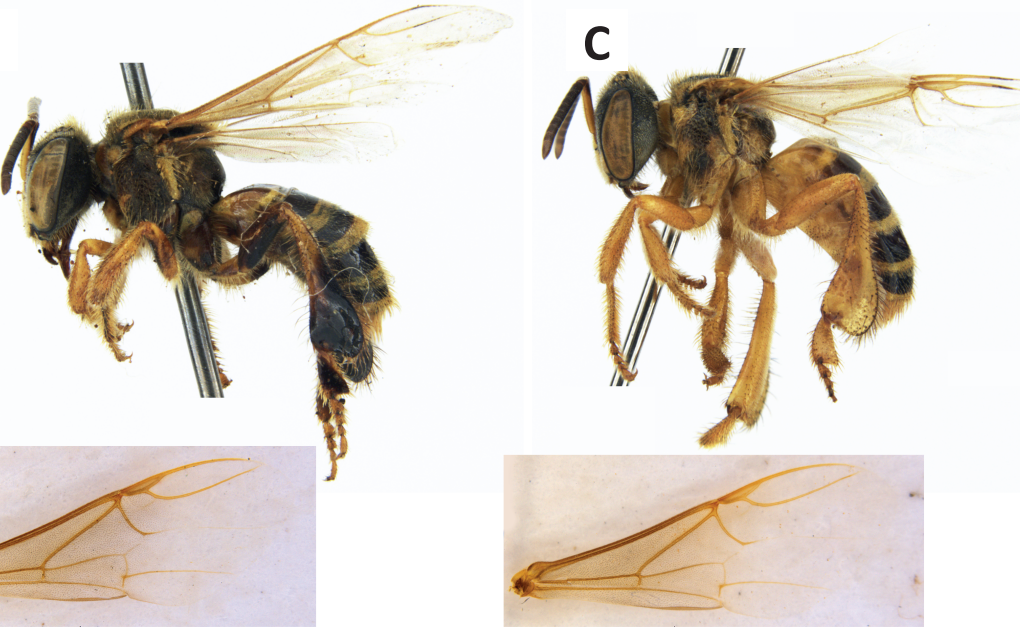

D

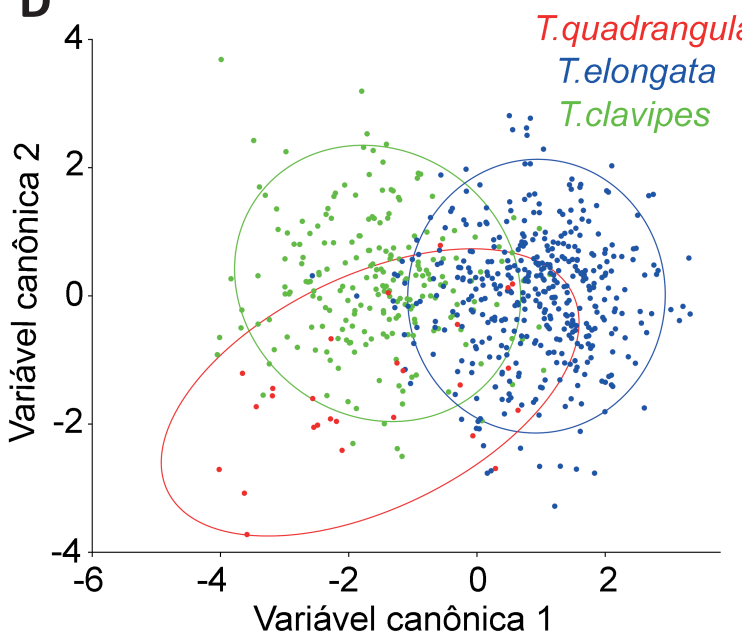

$\mathbf{E}$

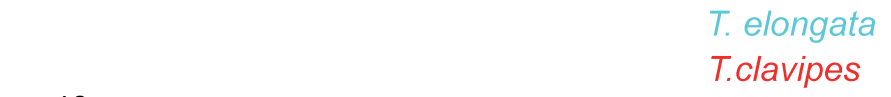

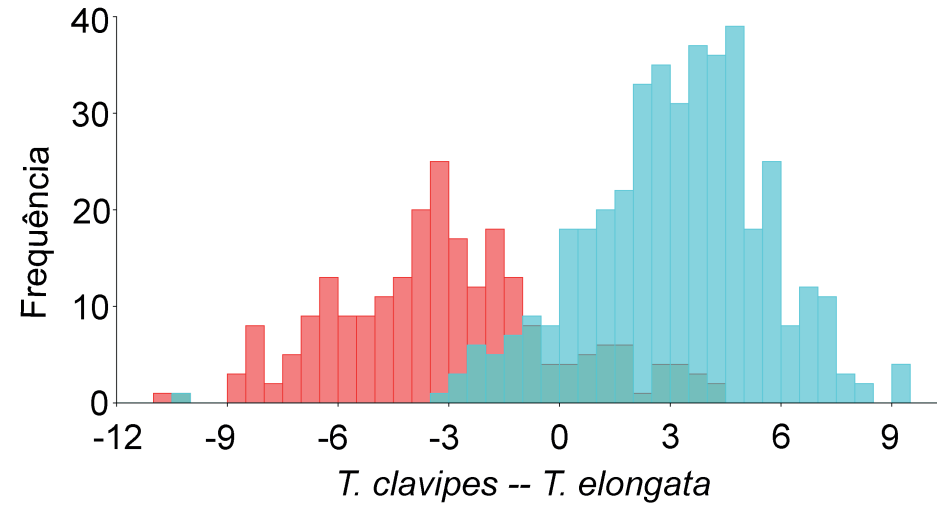

Figura 2. Vista lateral do corpo e asa de Tetragona clavipes (Fabricius, 1804) (A), T. elongata (Lepeletier \& Serville, 1828) (B) e T. quadrangula (Lepeletier, 1836) (C). Escalas de corpo e asa igualadas a $1 \mathrm{~mm}$. Análise canônica com o critério "Espécie" realizada com espécimes de T. quadrangula, T. elongata e T. clavipes, e com variância de 93,22\% (D). Análise de Função Discriminante com o critério "Espécie" com 14,83\% de sobreposição de T. clavipes com T. elongata e 9,70\% de sobreposição de T. elongata com T. clavipes (E).

\section{Agradecimentos}

A todas as instituições e curadores que nos enviaram espécimes: Isabel Alves-dos-Santos (CEPANN), Marcio Oliveira (INPA), Orlando Silveira (MPEG), Weyder Santana e Lucio Campos (UFV) e Eduardo Almeida (RPSP). ISS agradece ao apoio financeiro da bolsa pelo Conselho Nacional de Desenvolvimento Científico e Tecnológico (CNPq) - 01280.002501/2019-11, DSN agradece pelo suporte do Instituto Federal de Educação, Ciência e Tecnologia do Amazonas (IFAM) e ao Programa de Pós-Graduação em Entomologia do Instituto Nacional de Pesquisas da Amazônia (PPG-ENT). Esse estudo foi financiado em parte pela Coordenação de Aperfeiçoamento de Pessoal de Nível Superior - Brasil (CAPES) - Código de Financiamento 001, e FAPEAM-Programa POSGRAD.

\section{Contribuições dos autores}

ISS realizou as análises e escreveu o artigo. DSN e JSGT auxiliaram na execução das análises e escrita do artigo. IC e GSF auxiliaram na obtenção das amostras de T. elongata e escrita do artigo. MLO orientou todo o processo de análise de dados. Todos os autores leram o manuscrito e fizeram suas contribuições. MLO agradece ao CNPq-Brasil pela bolsa de produtividade (305150/2020-0)

\section{Referências}

Bookstein, F. L. (1991) Morphometric tools for landmark data. Geometry and Biology. New York: Cambridge University Press.

Camargo, J. M. F.; Pedro, S. R. M. (2013) Meliponini Lepeletier, 1836.
In: Moure, J. S., Urban, D.; Melo, G. A. R. (Eds.). Catalogue of Bees (Hymenoptera, Apoidea) in the Neotropical Region. http://www. moure.cria.org.br/catalogue. Acesso em: 22.v.2020.

Duarte, R.; Souza, J.; Soares, A. E. E. (2016) Nest architecture of Tetragona clavipes (Fabricius) (Hymenoptera, Apidae, Meliponini). Sociobiology, 63: 813-818. doi: 10.13102/sociobiology.v63i2.1019

Fabricius, J. C. (1804) Systema Piezatorum secundum ordines, genera, species, adjectis synonymis, locis, observationibus, descriptionibus. Brunsvigae: Reichard 1-439 pp.

Francoy, T. M.; Prado, P. R. R.; Gonçalves, L. S.; Costa, L. F.; Jong, D. (2006) Morphometric differences in a single wing cell can discriminate racial types. Apidologie, 37(1): 91-97. doi: 10.1051/apido:2005062

Francoy, T. M; Wittmann, D.; Drauschke, M.; Müller, S.; Steinhage, V. S.; Bezerra-laure, M. A. F.; Jong, D.; Gonçalves, L. S. (2008) Identification of Africanized honey bees through wing morphometrics: two fast and efficient procedures. Apidologie, 39(5): 488-494. doi: 10.1051/ apido:2008028

Francoy, T.; Wittmann, D.; Steinhage, V.; Drauschke, M.; Müller, S.; Cunha, D. R.; Nascimento, A. M.; Figueiredo, V.L.; Simões, Z. L. P.; Jong, D.; Arias, M. C.; Gonçalves, L. S. (2009) Morphometric and genetic changes in a population of Apis mellifera after 34 years of Africanization. Genetics and Molecular Research, 8(2): 709-717. doi: 10.4238/vol8-2kerr019

Kerr, W. E.; Sakagami, S. F.; Zucchi, R.; Portugal Araújo, V.; Camargo, J. M. F. (1967) Observações sôbre a arquitetura dos ninhos e comportamento de algumas espécies de abelhas sem ferrão das vizinhanças de Manaus, Amazonas (Hymenoptera, Apoidea). Atas do Simpósio sôbre a Biota Amazônica, 5: 255-309.

Monteiro, L. R.; Reis, S. F. (1999) Princípios de Morfometria Geométrica. 
Ribeirão Preto: Editora Holos.

Nogueira, D. S.; Ribeiro, C. F.; Oliveira, M. L. (2020) Taxonomia histórica de Nogueirapis Moure, 1953, Scaura Schwarz, 1938, Tetragona Lepeletier \& Serville, 1828 e Trigona Jurine, 1807 (Apidae: Meliponini). In: Oliveira-Junior, J. M. B.; Calvão, L. B. (Eds.). A Interface do Conhecimento sobre Abelhas 2. pp.63-77. Ponta Grossa: Atena Editora.

Nogueira, D. S.; Rasmussen, C.; Oliveira, M. L. (2021) A new species of Tetragona Lepeletier \& Serville, 1828 from "truncata group" and new distribution records of T. truncata Moure, 1971 (Hymenoptera: Apidae). Neotropical Entomology, 50: 68-77. doi: 10.1007/s13744020-00822-6

Oliveira, F. F.; Richers, B. T. T.; Silva, J. R.; Farias, R. C.; Matos, T. A. L. (2013) Guia ilustrado das abelhas "sem-ferrão" das reservas Amanã e Mamirauá, Amazonas, Brasil (Hymenoptera, Apidae, Meliponini). Tefé: Instituto de Desenvolvimento Sustentável Mamirauá.

Pedro, S. (2014) The stingless bee fauna in Brazil (Hymenoptera: Apidae). Sociobiology, 61: 348-354. doi: 10.13102/sociobiology. v61i4.348-354

Spinola. M. (1840) Observationes sur les apiaries meliponides. Annales des Sciences Naturelles, Zoologie et Biologie Animale, 13: 116-140. 